Formulation of indicators for the measurement of products of engineering research groups for their application in the SINFONI platform of the Cooperative University of Colombia

\title{
Formulación de indicadores para la medición de productos de los grupos de investigación de ingeniería para su aplicación en la plataforma SINFONI de la Universidad Cooperativa de Colombia
}

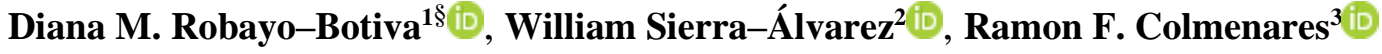 \\ ${ }^{1}$ Universidad Cooperativa de Colombia, Facultad de Ciencias Económicas, Administrativas y \\ Contables, Villavicencio, Colombia \\ ${ }^{2}$ Universidad Cooperativa de Colombia, Facultad de Ingeniería, Villavicencio, Colombia \\ ${ }^{3}$ Universidad Cooperativa de Colombia, Facultad de Ingeniería, Medellín, Colombia \\ §diana.robayo@campusucc.edu.co,william.sierraalv@campusucc.edu.co,ramon.colmenaresq@ucc.edu.co
}

Recibido: 7 de julio de 2020 - Aceptado: 19 de febrero de 2021

\begin{abstract}
The purpose of the research is to propose performance indicators for their implementation in the SINFONI research information system, and thus to measure the research groups initially in the engineering area. The methodology includes the characterization of the University's research professors and the research groups to which they belong, the


identification of the contribution of the engineering research groups to the regions in coherence with the Sustainable Development Goals (SDGs), the departmental development plans and lines of research from the projects registered on the GrupLAC and SINFONI platform, and additionally, conducting interviews with the personnel in charge of SINFONI in order to know the system and its gear not only with the plan National Strategic 2013 - 2022 "Navigating Together" (PEN) but also with development plans and the SDGs through indicators. The initial phases of the TOGAF enterprise architecture model were used and applied to a higher education institution. Among the conclusions are that the development of projects carried out by the engineering research groups that contribute to the departmental development plans is not very significant, however, the projects are related to the SDGs, on the other hand, with respect to SINFONI, despite its robustness and its interaction with other university systems, it does not have indicators to demonstrate compliance with the strategic research objectives defined in the PEN, nor its contribution to departmental development plans, so the contribution is a proposal of indicators. The inclusion of the indicators in SINFONI will allow the realization of measurements to determine the contribution of the university to the regions, SDGs and finally demonstrate compliance with the PEN (research strategic axis) and its mission.

Keywords: Indicators, Investigation, Measurement, Model, SINFONI, TOGAF.

\section{Resumen}

La investigación tiene como fin la proposición de indicadores de desempeño para su implementación en el sistema de información para la investigación SINFONI, y así realizar medición de los grupos de investigación inicialmente del área de ingeniería. La metodología contempla la caracterización de los profesores investigadores de la Universidad y de los grupos de investigación a los que pertenecen, la identificación del aporte de los grupos de investigación de ingeniería a las regiones en coherencia con los objetivos de desarrollo sostenible (ODS), los planes de desarrollo departamentales y las líneas de investigación a partir de los proyectos registrados en la plataforma del GrupLAC y SINFONI, y adicionalmente, la realización de entrevistas al personal encargado de SINFONI con el fin de conocer el sistema y su engranaje no solo con el plan Estratégico Nacional 2013 - 2022 "Navegando Juntos" (PEN) sino también con los planes de desarrollo y los ODS mediante indicadores. Se utilizó las fases iniciales del modelo de arquitectura empresarial TOGAF aplicándolo a una institución de educación superior. Dentro de las conclusiones se encuentran que el desarrollo de proyectos realizados por los grupos de investigación de ingeniería que aportan a los planes de desarrollo departamentales es poco significativo, no obstante, los proyectos se relacionan con los ODS, de otro lado, respecto a SINFONI, a pesar de su robustez y su interacción con otros sistemas de la universidad, no cuenta con indicadores para demostrar el cumplimiento de los objetivos estratégicos de investigación definidos en el PEN, ni tampoco su aporte a planes de desarrollo departamentales por lo que la contribución es una propuesta de indicadores. La inclusión de los indicadores en SINFONI permitirá la realización de mediciones para determinar el aporte de la universidad a las regiones, ODS y finalmente demostrar el cumplimiento del PEN (eje estratégico investigación) y su misión.

Palabras clave: Indicadores, Investigación, Medición, Modelo, SINFONI, TOGAF.

\section{Introducción}

La misión de la Universidad Cooperativa de Colombia relaciona el buscar mejoramiento de la calidad de vida de las comunidades, por tanto, es su interés mediante los grupos de investigación aportar a las regiones del país a partir de proyectos que satisfagan necesidades reales. No obstante, el desarrollo de proyectos debe ser no solo, en consonancia con el plan Estratégico Nacional (PEN) "Navegando Juntos" 2013 - 2022(1) sino también con los planes de desarrollo departamentales, los Objetivos de Desarrollo Sostenible (ODS), de tal manera, que esta alineación permita proyectos que contribuyan a las diversas regiones del país y conduzcan así al cumplimiento de la misión de la institución.

En este punto, es importante acotar que los ODS se definen en la Agenda 2030 de las Naciones Unidas $^{(2)}$ y fueron adoptados por el país para un desarrollo sostenible a largo plazo, asimismo, en el documento de la Agenda 2030 y los objetivos de desarrollo sostenible: Una oportunidad para 
América Latina y el Caribe $^{(3)}$ una de las prioridades de la CEPAL (Comisión Económica para América Latina y el Caribe) es potenciar el análisis de los medios de implementación de la Agenda 2030 a nivel regional y apoyar la integración de los ODS en los planes nacionales de desarrollo y en los presupuestos, de ahí la importancia de la contextualización de los ODS en las regiones a partir de los planes de desarrollo departamentales y municipales aportando a su vez al plan nacional de desarrollo.

Ahora bien, para validar sí los proyectos desarrollados por grupos de investigación además de beneficiar a comunidades, regiones se encuentran efectivamente alineados con ODS, planes de desarrollo departamentales y PEN (eje estratégico de investigación), se toma como caso específico los proyectos ejecutados por los grupos de investigación de ingenierías, y asimismo, el soporte suministrado por la plataforma SINFONI para evidenciar si existen indicadores que permitan medir los resultados del aporte de la investigación en aspectos ya mencionados como comunidades, planes, ODS, entre otros.

\section{Metodología}

¿El sistema SINFONI permite generar informes y/o reportes que evidencien el impacto de las investigaciones en la región, basados en aspectos como proyectos por región, personas y empresas beneficiadas, planes de desarrollo departamentales beneficiados, productos generados que permitan a su vez la toma de decisiones?

Inicialmente se realizó un instrumento con base al Modelo de Medición de grupos de investigación, desarrollo tecnológico o de innovación y de reconocimiento de investigadores del sistema nacional de ciencia, tecnología e innovación año $2018^{(4)}$, el cual fue validado por un Profesor Investigador categorizado como Investigador Asociado, con amplia experiencia en el desarrollo de instrumentos estadísticos para la caracterización de los profesores investigadores de la Universidad Cooperativa de Colombia y de los grupos de investigación que lideran.

Adicionalmente, se recopiló la información de los planes de desarrollo departamentales donde se encuentran los campus a los cuales pertenecen los grupos de investigación, también se consolido información registrada en el GrupLAC y el sistema SINFONI, lo anterior para identificar programas y proyectos de los planes de desarrollo departamentales a los cuales podrían apuntar los grupos de investigación de ingeniería, asimismo, se identificó el aporte de los grupos a las regiones en coherencia con los objetivos de desarrollo sostenible, los planes de desarrollo departamentales y a las líneas de investigación, finalmente, se realizaron entrevistas con el personal encargado de la plataforma SINFONI con el fin de conocer los servicios que ofrece este sistema, su alineación con el cumplimiento de los objetivos estratégicos de investigación del plan estratégico nacional (PEN), así como también con los planes de desarrollo departamentales, los ODS, igualmente, conocer los posibles indicadores de la plataforma utilizados para medir el aporte de los proyectos desarrollados por los grupos de investigación al PEN, planes de desarrollo, comunidades, ODS. La Figura 1 muestra una representación esquemática del desarrollo de la investigación.

\section{Resultados y discusión}

A continuación, se relacionan los resultados más representativos de la investigación:

\subsection{Caracterización de los profesores investigadores de la Universidad Cooperativa de Colombia y de los grupos de investigación que lideran}

De acuerdo con la encuesta de caracterización de los profesores investigadores de la universidad 

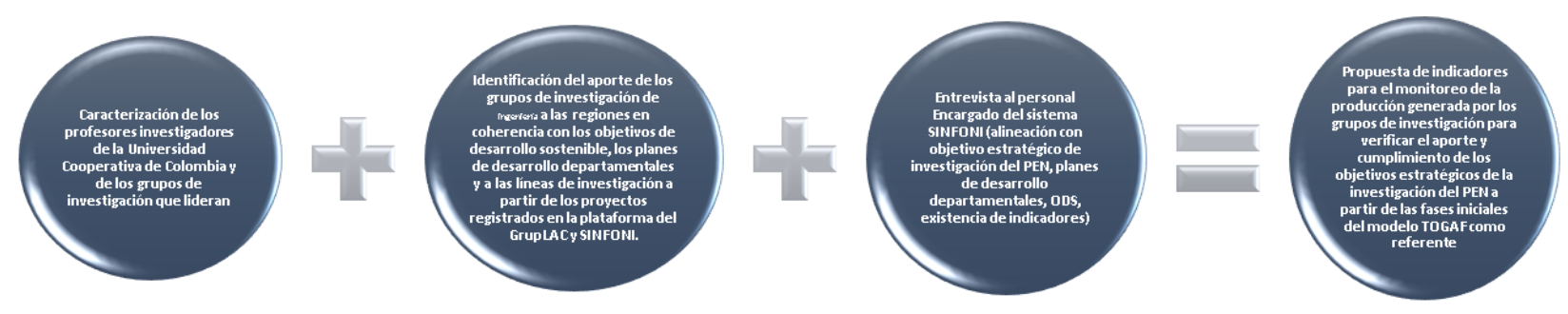

Figura 1. Representación esquemática del desarrollo de la investigación

Cooperativa de Colombia, se obtuvo los siguientes resultados generales, en cuanto a proyectos de investigación realizados con otros campus:

- Proyectos identificados: 16

- Campus vinculados: 10

- Grupos identificados: 13

En la Tabla 1 se relacionan en número de grupos y campus vinculados en el desarrollo de proyectos de investigación de la Universidad.

Se destaca lo siguiente acorde a la información consolidada:

- El campus Medellín cuenta con mayor número de proyectos que involucran otros campus de la Universidad Cooperativa de Colombia (13 proyectos teniendo en cuenta proyectos con información incompleta).

- Dos grupos de investigación (de acuerdo con la información registrada) contemplan el mayor número de proyectos multicampus

- Desarrollo de Proyecto de investigación con universidades externas.

3.2. Identificación del aporte de los grupos de investigación de ingeniería a las regiones en coherencia con los objetivos de desarrollo sostenible, los planes de desarrollo departamentales y a las líneas de investigación a partir de los proyectos registrados en la plataforma del GrupLAC y SINFONI.
Del material recopilados de planes de desarrollo de los departamentos del Meta ${ }^{(5)}$, Tolima ${ }^{(6)}$, Magdalena $^{(7)}$ Valle del Cauca ${ }^{(8)}$, Santander ${ }^{(9)}$, Cundinamarca $^{(10)}$, Antioquia ${ }^{(11)}$, Nariño ${ }^{(12)}$, Arauca $^{(13)}$ y de la plataforma GrupLAC y SINFONI se realizó un análisis por nueve departamentos, que como se indicó anteriormente son aquellos donde hay un campus de la universidad y por tanto hay un grupo de investigación en el área de ingeniería. El análisis relaciono factores como programas y proyectos de los planes de desarrollo departamentales y su relación con los ODS y las líneas de investigación de ingeniería, además, se contempló la producción reportada en las plataformas antes mencionadas para determinar posible relación con entidades gubernamentales, ODS, desarrollo de proyectos multicampus, visibilidad del impacto de los proyectos en la comunidad, lo cual fue muy fructífero para determinar el estado de los grupos de ingenierías de la Universidad Cooperativa de Colombia (2019). A continuación, se relacionan apartes de los resultados del análisis realizado en uno de los nueve departamentos registrados en el proyecto:

\subsubsection{Departamento de Nariño}

Acorde al análisis del plan de desarrollo del Departamento de Nariño (Figuras 2, 3, 4) se puede apreciar que las metas del plan apuntarían en especial a la sub - línea de investigación de Tecnologías de la información y telecomunicaciones, seguido por estructuras, materiales y suelos, del mismo modo que 
Tabla 1. Proyectos Multicampus

\begin{tabular}{|c|c|c|}
\hline Proyecto & $\begin{array}{c}\text { Grupos } \\
\text { vinculados }\end{array}$ & $\begin{array}{c}\text { Campus } \\
\text { vinculados }\end{array}$ \\
\hline $\begin{array}{l}\text { Bienestar psicológico, calidad de vida y apoyo social de población víctima } \\
\text { del conflicto armado en Colombia }\end{array}$ & 3 & 5 \\
\hline Filiación socioafectiva & 2 & 2 \\
\hline $\begin{array}{l}\text { Paz con enfoque territorial y solidario: prácticas y percepciones } \\
\text { comunitarias. Insumos para una agenda social }\end{array}$ & 2 & 2 \\
\hline $\begin{array}{l}\text { Desarrollo de portafolios electrónicos para la evaluación del aprendizaje por } \\
\text { competencias en entornos virtuales }\end{array}$ & 2 & 2 \\
\hline Acelerando la Energía Marina en Colombia & 2 & 2 \\
\hline $\begin{array}{l}\text { Efecto de Lactobacillus rhamnosus y Bifidobacterium longum en niños de } 3 \\
\text { a } 5 \text { años de Villavicencio y Pasto. }\end{array}$ & 2 & 2 \\
\hline $\begin{array}{l}\text { Evaluación espacial y temporal de la calidad del agua de quebrada Las } \\
\text { Delicias, Cerros Orientales de Bogotá }\end{array}$ & 2 & 2 \\
\hline Evaluación de la calidad del Agua en Urabá & 2 & 2 \\
\hline $\begin{array}{l}\text { Las nuevas fuentes de productividad en la economía del conocimiento: } \\
\text { análisis del papel de las instituciones en el uso de factores productivos y } \\
\text { desarrollo económico }\end{array}$ & 1 & 2 \\
\hline $\begin{array}{l}\text { Evaluación del Uso De La Biomasa del Departamento Del Huila Evaluación } \\
\text { del Uso de la Biomasa del Departamento del Huila en la Producción de } \\
\text { Biocombustible Densificado (Pellet) de Alto Poder Calorífico. }\end{array}$ & 1 & 1 \\
\hline Estrés y Riesgo de Psicosis en Población General & 2 & 2 \\
\hline $\begin{array}{l}\text { Determinación mediante análisis metagenómico de la relación entre los } \\
\text { periodontopatógenos y la enfermedad coronaria }\end{array}$ & 1 & 2 \\
\hline Validación del inventario de personalidad PAI-A & 1 & $\begin{array}{l}2 \\
1 \text { y grupo }\end{array}$ \\
\hline $\begin{array}{l}\text { La expresión de la anterioridad al momento del habla en la variedad } \\
\text { colombiana del español }\end{array}$ & 1 & $\begin{array}{l}\text { de } \\
\text { universidad } \\
\text { Externa. }\end{array}$ \\
\hline Desarrollo de una red acelero métrica de bajo costo para movimiento fuerte & 1 & 2 \\
\hline $\begin{array}{l}\text { Tensiones Jurídicas en la aplicación de la justicia indígena y la justicia } \\
\text { ordinaria: escenarios y prácticas jurídicas en la comunidad indígena de } \\
\text { Dojura en el municipio de Chigorodó y Las Plamas en el municipio de } \\
\text { Apartadó }\end{array}$ & 1 & 2 \\
\hline $\begin{array}{l}\text { Análisis socio jurídico de la reivindicación política de los DESC en } \\
\text { contextos de ciudad a partir de un grupo de imágenes fotográficas en la Calle } \\
\text { 5a. de la ciudad de Cali }\end{array}$ & 2 & 2 \\
\hline
\end{tabular}

apuntarían a varios ODS, por tanto, en esta región también se presentan oportunidades de trabajo con impacto social, no solo para el grupo del campus Pasto sino a su vez, para todos los grupos de ingenierías de la Universidad Cooperativa de Colombia.

De otro lado, según la información de proyectos vinculados con el grupo ESLINGA en el
GrupLAC (campus Pasto) se encuentran relacionados los trabajos entre el 2017 - 2015:

Estudio para la implementación de un laboratorio de testing de software para los desarrollos de aplicativos móviles en la universidad cooperativa de Colombia - sede Popayán

Diseño e implementación de un prototipo tecnológico y empresarial para el 
aprovechamiento energético de aceite de cocina usado en el municipio de Pasto - Junta de Acción Comunal - barrio Obrero. SERPRO INGENIERIA SAS.

Fundamentos para la implementación de un modelo de gestión del conocimiento como herramienta del valor en pymes dos casos de estudio

Respecto a proyectos de 2018 en la plataforma institucional:

Evaluación de la relación entre los riesgos ocupacionales causado por la incidencia de la radiación solar uva y uvb y el disconfort térmico en las áreas de trabajo de la sede de Pasto de la universidad Cooperativa de Colombia.

Identification of knowledge gaps in the academia and capacity building for aquatic renewable energy in Colombia - AQUATERA. COTECMAR. HERIOT WATT UNIVERSITY.

Información tomada de la Pagina de Grupos y Semilleros del micrositio de Investigación del Sitio oficial de la Universidad Cooperativa de Colombia. ${ }^{(14)}$

La información registrada en SINFONI y GrupLAC (proyectos investigación y desarrollo) demuestra que al igual otros grupos de investigación del campus Pasto tiene una producción significativa de proyectos, con un total de 12 entre el 2015 - 2018, correspondiente al desarrollo de 3 proyectos anuales, no obstante, comparado con uno de los grupos de investigación de ingeniería de Pasto, esto equivale a la mitad de los proyectos anuales desarrollados por este último grupo. Cabe mencionar que ESLINGA ha desarrollado un mayor número de proyectos que vinculan campus de la Universidad Cooperativa de Colombia, universidades, corporaciones, entidades públicas y privadas, por lo que a través del desarrollo de las investigaciones buscan aportar a necesidades de la comunidad.

Los proyectos ejecutados hasta el momento apuntan a su vez a ODS como industria, innovación e infraestructura, energía asequible y no contaminante, acción por el clima, Educación de calidad, salud y bienestar, producción y consumo responsables, por lo que, la mayor parte de estos trabajos aportan al cuidado del medio ambiente y energías renovables. En cuanto a la relación de los proyectos con las metas del plan de desarrollo del departamento de Nariño, no hay un aporte puntual al mismo, más sin embargo, se evidencia apoyo en el desarrollo de proyectos con corporaciones y Junta de Acción Comunal, acorde a lo anteriormente expuesto, el campo de trabajo e investigación del grupo de ingenierías en el departamento de Nariño es amplio toda vez que no se ha explotado las oportunidades en la región a través del desarrollo de proyectos que apunten al plan de desarrollo departamental.

Finalmente, de acuerdo con el análisis realizado a la información de los grupos de investigación (a partir de la información del GrupLAC y la plataforma institucional), se puede concluir:

No hay proyectos de investigación realizados por los grupos que aporten de manera puntual a los planes de desarrollo departamentales

Los proyectos de investigación desarrollados en los últimos años tienen como fin cooperar con el medio ambiente.

Los proyectos de investigación apuntan a los objetivos de desarrollo sostenible

La evidencia de trabajos multicampus entre los grupos de investigación de ingenierías es poco significativa. 


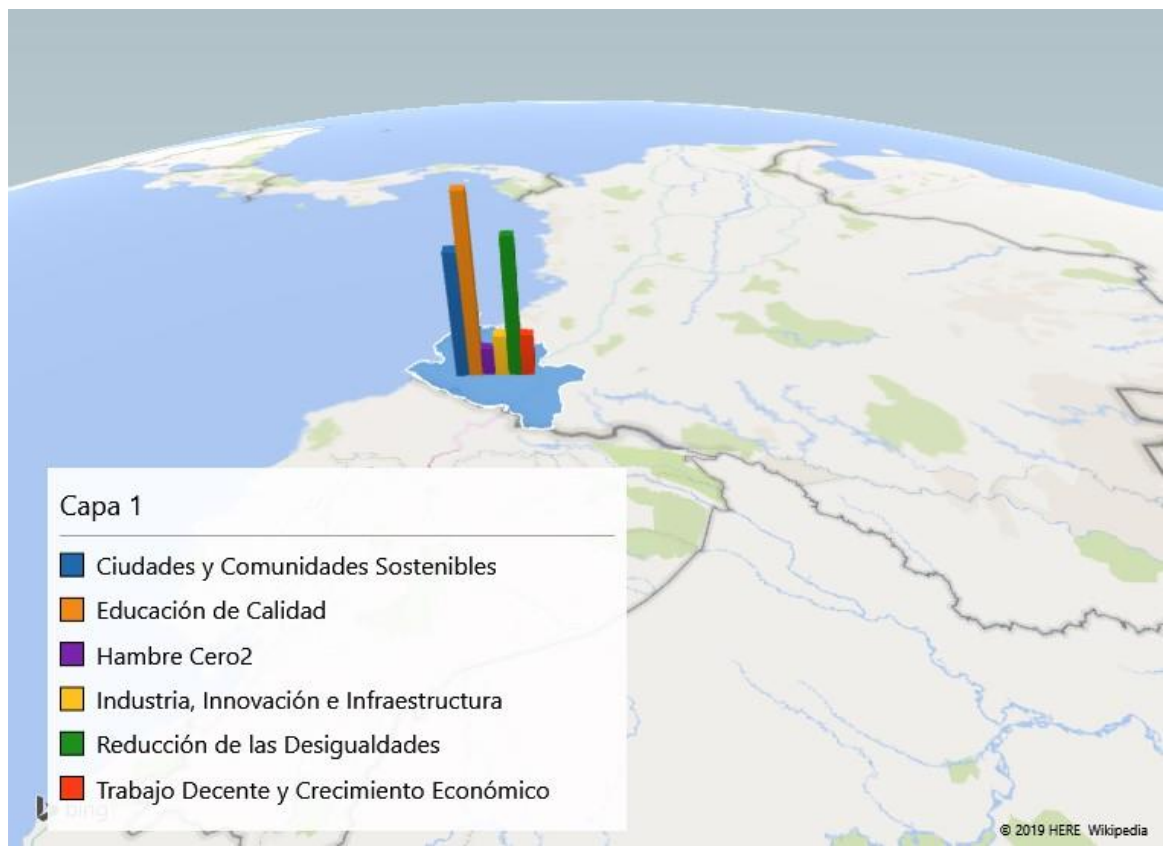

Figura 2. Relación Metas Planes de Desarrollo- Líneas de Investigación- ODS Departamento de Nariño

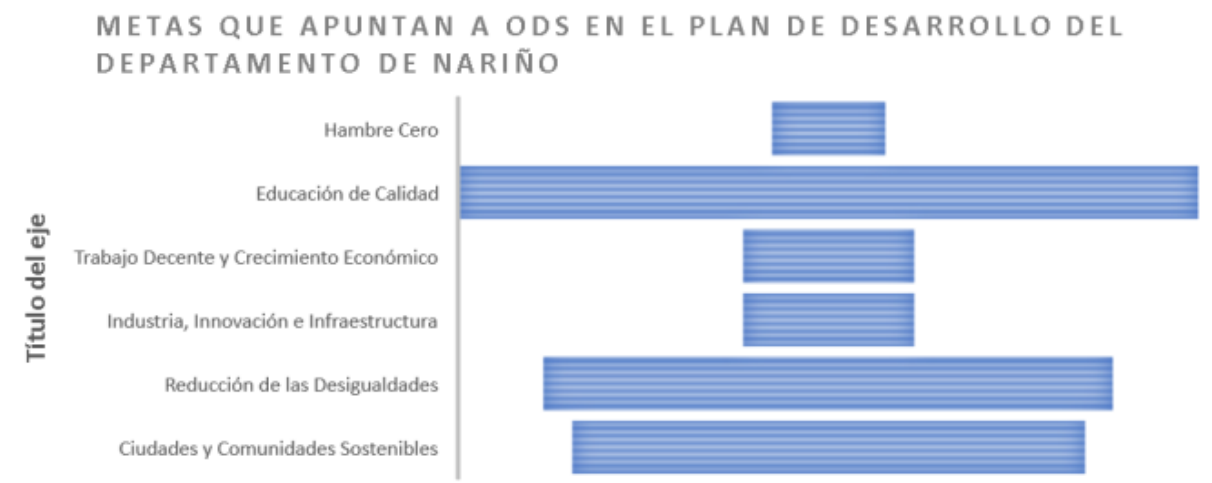

Figura 3. Metas que apuntan a ODS en el plan de desarrollo del departamento de Nariño

Sub - líneas de investigacíon que apuntan a las metas del plan de desarrolloy a los ODS

TECNOLOGIAS DE LA INFORMACION Y DE LAS TELECOMUNICACIONES

ESTRUCTURAS MATERIALES Y SUELOS

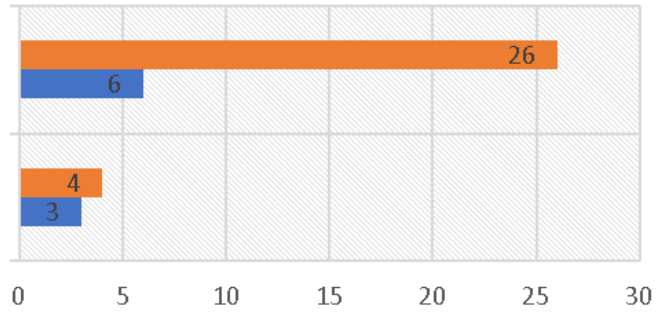

- Número de metas del plan de desarrollo relacionadas con la sub - línea

- Número de ODS relacionados con las sub - líneas

Figura 4. Sub-líneas de Investigación que apuntan a las metas de desarrollo y los ODS departamento de Nariño 
La ejecución de proyectos con participación de entidades públicas y privadas no es totalmente significativa.

La Universidad cuenta con grupos que pueden aportar a las necesidades del país, toda vez que la institución hace presencia en diferentes regiones del país, pero el análisis de la información demuestra que para visibilizar el impacto social de la institución a través de los proyectos desarrollados y así demostrar el cumplimiento de los objetivos estratégicos de investigación establecidos en el PEN 2013 - 2022, es necesaria una herramienta o en este caso, alinear el sistema con que cuenta la institución (SINFONI), para que sea un aliado estratégico permitiendo que los grupos puedan conocer de primera mano necesidades de las diferentes regiones a partir de la consulta de los planes de desarrollo y así impactar en empresas, personas, comunidades con productos pertinentes, a su vez el sistema debe ser un espacio de encuentro de los grupos que desean trabajar en equipo para solventar dichas necesidades. Igualmente, es fundamental el apoyo de las directivas de la institución estableciendo las alianzas necesarias con las distintas gobernaciones para que se dé vía libre a los grupos y apunten directamente a los planes con apoyo de las entidades públicas, logrando también estrechar la relación Universidad Empresa - Estado.

\subsection{Propuesta de indicadores para el monitoreo de la producción generada por los grupos de investigación para verificar el aporte y cumplimiento de los objetivos estratégicos de la investigación del PEN a partir de las fases iniciales del modelo TOGAF como referente}

Acorde a los modelos de arquitecturas consultados, se utilizará TOGAF $^{(15-23)}$ en la fase preliminar y en la visión de la arquitectura como referencia. En este punto, es importante reiterar el uso del modelo TOGAF como referente en el desarrollo del proyecto, ya que aun cuando este modelo es usado para la alineación de los objetivos estratégicos de las organizaciones con las TI (Tecnologías de la Información), este puede ser utilizado desde otras perspectivas en las instituciones de educación superior (IES) puesto que sirve de guía en la revisión de la alineación de algo tan especifico pero fundamental en las IES como es la investigación (es una las funciones sustantivas de una IES) con las TI que la universidad provee para evidenciar el cumplimiento de dicha función sustantiva, esta última plasmada en objetivos relacionados en planes estratégicos, adicionalmente, respecto a lo anterior, cabe destacar lo mencionado por Saboya et al. ${ }^{(24)}$ respecto al uso de la AE TOGAF 9.0 para publicaciones científicas en relación con el desarrollo del proyecto en cuestión:

Identificar la estrategia de la organización y obtener su compromiso y participación: En este aspecto se diseñó un instrumento para la identificación de los grupos de investigación, líneas de investigación, clasificación de los grupos según GrupLAC, cruce de las líneas de investigación con los planes de desarrollo regionales.

Identificar (entender) la situación actual y diseñar (proponer) la situación futura en relación a los dominios de la arquitectura de negocios, sistemas de información y tecnología: En este aspecto, se revisaron los procedimientos establecidos para los procesos de investigación por la dirección de Investigación a través de SINFONI (sistema implementado por la universidad para la gestión de la investigación) y la su alineación con el cumplimiento de los objetivos estratégicos de investigación definidos en el PEN 2013 - 2022.

Implementación de la transformación, en cuanto a este aspecto, al revisar los objetivos estratégicos de investigación del PEN 2013 - 2023 y teniendo en cuenta la información obtenida de SINFONI (sistema de información implementado por la 
universidad para la gestión de la investigación) a partir de reuniones con el personal encargado del sistema, se evidencio que a pesar de la robustez del mismo y su interacción con otros sistemas de la universidad, este no cuenta con indicadores claves que permitan demostrar el cumplimiento de los objetivos estratégicos de investigación definidos en el PEN 2013 - 2022, por lo que se estableció en el proyecto la propuesta de indicadores ${ }^{(25)}$. Se relaciona entonces, de manera general el análisis de la información con las fases iniciales del modelo TOGAF como referente para la generación de los indicadores propuestos:

\subsection{Fase Preliminar}

Para dar cumplimiento a lo dispuesto en la misión y visión institucional ${ }^{(26)}$ : "En el año 2022 seremos una universidad de docencia con investigación..." la Universidad Cooperativa de Colombia establece los procesos y mecanismos que orientan el desarrollo de la investigación bajo los siguientes parámetros normativos:

Proyecto Institucional. Acuerdo 147 de 2013.

Estatuto General y Estructura Administrativa de la Investigación en la Universidad Cooperativa de Colombia. Acuerdo Superior $N^{\circ} 173$ del 20 de marzo de 2014

Resolución Rectoral No 840 de noviembre de 2014 por medio de la cual se establece los lineamientos para la conformación y desarrollo del trabajo en los semilleros de investigación para la Universidad Cooperativa de Colombia.

Plan Estratégico Nacional (PEN) 2013-2022. Navegando Juntos

Respecto al plan estratégico nacional PEN del a UCC, en su carta de navegación proyecta la misión, visión, principios y valores a un objetivo primordial, "llevar a la docencia a reconocer y desarrollar una investigación que imparte y transforme lo social". Su modelo educativo enfocado en las competencias que dan herramientas a los estudiantes para enfrentar las transformaciones del siglo XXI, de una forma integral: Academia, Gobierno y Sociedad, en sincronía con los Objetivos de Desarrollo Sostenible (ODS).

En las Apuestas Estratégicas Institucionales del Plan Estratégico Nacional, la segunda apuesta "Una Comunidad Académica y Científica, Sólida y Visible Nacional e Internacionalmente".

Agrupa los investigadores de todas las áreas del conocimiento para enfocar sus saberes en actividades de investigación, desarrollo e innovación en beneficio de la sociedad. Se destacan los objetivos estratégicos: "consolidar la investigación en todos los campos del saber existentes en la universidad, para que articulada a la docencia y la proyección social se constituya en una parte sustancial de la cultura académica." y "realizar investigación pertinente que genere impactos nacionales", este objetivo estratégico es importante pues hace parte de las funciones sustantivas de una institución de educación superior, el interés del proyecto es determinar si los grupos de investigación de ingenierías están apuntando al beneficio de las comunidades a partir de los ODS, planes de desarrollo departamentales, evidenciando una alineación con los objetivos estratégicos de investigaciones definidos en el PEN 2013 - 2022, por lo que se realiza lo siguiente:

Realización de una caracterización de los profesores investigadores de la Universidad Cooperativa de Colombia y de los grupos de investigación que lideran

Identificación del aporte de los grupos de investigación de ingeniería a las regiones en coherencia con los objetivos de desarrollo sostenible, los planes de desarrollo departamentales y a las líneas de investigación a partir de los proyectos registrados en la plataforma del GrupLAC y SINFONI. 
Reconocimiento de la Estructura de la investigación en la Universidad Cooperativa de Colombia

Caracterización de los Grupos de investigación de ingeniería por líneas de investigación.

\subsection{Visión de Arquitectura}

Teniendo en cuenta la necesidad de realizar seguimiento a la producción generada por los grupos de investigación para verificar el aporte y cumplimiento de los objetivos estratégicos de la investigación del PEN 2013 - 2022:

"Consolidar la investigación en todos los campos del saber existentes en la universidad, para que articulada a la docencia y la proyección social se constituya en una parte sustancial de la cultura académica."

"Realizar investigación pertinente que genere impactos nacionales"

Alineado a su vez con ODS y planes de desarrollo departamentales. Ahora bien, partiendo del hecho que desde el 2017 la Universidad Cooperativa de Colombia implemento el sistema de información SINFONI (orientado solo a investigación), el cual no se tuvo acceso como tal, pero se pudo conocer a partir de las reuniones del personal encargado, con el fin de determinar sí este sistema robusto tiene indicadores que permitan realizar seguimiento a la producción generada por los grupos de investigación para comprobar su alineación con los objetivos estratégicos de investigación,

Para el análisis de este aspecto, como se indicó anteriormente, se realizó entrevista a personal de apoyo encargado de la plataforma SINFONI para el conocimiento de este. Luego, a partir de la información obtenida mediante las entrevistas la cual permitió entender cómo funciona SINFONI.
Sin embargo, se evidencia que aun cuando este sistema es robusto para la gestión de la investigación en la Universidad, no está alineado en su totalidad con los objetivos estratégicos de investigación establecidos en el PEN 2013 2022, lo cual fue validado por el personal encargado, por ende, se construyeron una serie de indicadores que fueron validado por el personal de SINFONI, los cuales podrían ser utilizados no solo para los grupos del área de ingeniería sino también para grupos de investigación de otras áreas del conocimiento. Los indicadores propuestos permiten obtener información que permita medir con mayor claridad el impacto de la investigación en las regiones, además, de permitir una toma de decisiones eficientes $y$ asimismo propender al cumplimiento de los objetivos estratégicos de la investigación. Se registran algunos de los indicadores propuestos más representativos en la Figura 5.

\section{Conclusiones}

Con el desarrollo de la investigación se estableció que:

No hay trabajo colaborativo para el desarrollo de proyectos multicampus. La investigación multicampus sirve para proyectos de alto impacto en las regiones y esta debe ser la prioridad para los líderes de los grupos de investigación

El acercamiento con las entidades gubernamentales (alcaldías y gobernaciones) es muy poco, las investigaciones no están apuntando a los planes de desarrollos y las entidades no se han apoyado en la academia para ejecutar los planes.

Las líneas de investigación en los grupos de ingenieras (desarrollo de software, aguas y medio ambiente, energías renovables, estructuras, tecnologías de la información y comunicaciones) tienen un gran potencial para desarrollar proyectos de gran envergadura que pueden 
FIN (ANUAL) - EN RELACION CON EL OBJETIVO ESTRATEGICO

INVESTIGACION DEL PEN 2013-2022

CONTRIBUIR A LAS RE GIONE S DEL PAIS MEDIANTE EL DE SARR OLLO DE PROYECT OS DE
APROPLACION SOCIAL PARABENEFICIAR A LAS COMUNIDADES ALCANZANDOUN IMPACT ORE GIONAL

Indicador de eficacia (efectos sociales y económicos)

\begin{tabular}{|c|}
\hline $\begin{array}{l}\text { (\# de proyectos ejecutados que benefician a la región norte en el periodo titotal de proyectos ejecutados que } \\
\text { benefician a las regiones del pais en el periodo t) } \times 100 \%\end{array}$ \\
\hline $\begin{array}{l}\text { (\# de proyectos ejecutados que aportan al cambio climático en el periodo títotal de proyectos ejecutados en el } \\
\text { periodo t) } x 100 \%\end{array}$ \\
\hline Propósito \\
\hline $\begin{array}{l}\text { Aportar a la sociedad con proyectos que tributen a los objetivos de desarrollo sostenible favoreciendo } \\
\qquad \text { a las distintas regiones del pais }\end{array}$ \\
\hline Indicador de eficacia \\
\hline $\begin{array}{l}\text { (Número de proyectos que tributan a los planes departamentales del país en el periodo títotal de proyectos } \\
\text { ejecutados en el periodo t) } x 100 \%\end{array}$ \\
\hline Componente-Productos \\
\hline $\begin{array}{l}\text { 1. Productos de generación de nuevo conocimiento } \\
\text { 2. Productos de innovación } \\
\text { 3. Productos de apropiación social de conocimiento }\end{array}$ \\
\hline Indicadores de calidad \\
\hline $\begin{array}{l}\text { (Regiones beneficiadas por el producto en el periodo titotal de regiones de aprendizaje definidas por la } \\
\text { institución en el periodo t) } x 100 \%\end{array}$ \\
\hline
\end{tabular}

Figura 5. Propuesta de indicadores para monitoreo de la producción generada por los grupos de investigación para verificación del aporte y cumplimiento de los objetivos estratégicos de la investigación del PEN

apuntar a los objetivos de desarrollo sostenible ODS en beneficio de las comunidades (se puede apreciar en los cuadros comparativos) y así dar su aporte a la sociedad; es importante aunar esfuerzos para el cumplimiento de los objetivos propuestos en el eje estratégico de investigación del PEN.

La primicia de la Universidad Cooperativa de Colombia expuesta en el Plan Estratégico Nacional PEN "llevar a la docencia a reconocer y desarrollar una investigación que imparte $\mathrm{y}$ transforme lo social" es de tipo incluyente ya que busca acercar la investigación a la solución de problemas reales de las comunidades y que puedan ser sostenibles en el tiempo, como lo manifiesta la agenda 2030 expuesta en Objetivos de Desarrollo Sostenible ODS. La UCC cuenta con una plataforma para el manejo y administración de la información de investigación SINFONI, la cual mediante convocatorias administra proyectos de investigación ejecutados por los diversos grupos de investigación que tiene la universidad, todos los procesos de adjudicación, control, manejo, asesoría, revisión 
y finalización de los proyectos los tienen bien documentados, sin embargo, este sistema no realiza un seguimiento en cuanto a impacto de las investigaciones en las regiones y así corroborar su alineación con los objetivos estratégicos de investigación definidos en el PEN.

\section{Declaración de financiación}

Este trabajo de investigación se realizó sin ningún financiamiento de ninguna entidad.

\section{Referencias}

(1) Universidad Cooperativa de Colombia. Plan Estratégico [Internet]. 2017 [citado el 19 de marzo de 2021]. Disponible en: https://www.ucc.edu.co/institucion/Pagina s/plan-estrategico.aspx

(2) Naciones Unidas. Objetivos de Desarrollo Sostenible (ODS) [Internet]. Cepal.org. 2017 [citado el 19 de marzo de 2021]. Disponible en: https://www.cepal.org/es/temas/agenda2030-desarrollo-sostenible/objetivosdesarrollo-sostenible-ods

(3) Naciones Unidas. La Agenda 2030 y los Objetivos de Desarrollo Sostenible: una oportunidad para América Latina y el Caribe. Objetivos, metas e indicadores mundiales [Internet]. Cepal.org. Santiago; 2019 [citado el 19 de marzo de 2021]. Disponible en: https://www.cepal.org/es/publicaciones/40 155-la-agenda-2030-objetivos-desarrollosostenible-oportunidad-america-latinacaribe

(4) COLCIENCIAS. Modelo de Medición de Grupos de Investigación, Desarrollo Tecnológico o de Innovación y de Reconocimiento de Investigadores del Sistema Nacional de Ciencia, Tecnología E
Innovación, AÑO 2018 [Internet]. Gov.co. 2018 [citado el 6 de diciembre de 2019]. Disponible en: https://minciencias.gov.co/sites/default/fil es/upload/convocatoria/4._anexo_1._docu mento_conceptual_del_modelo_de_recon ocimiento_y_medicion_de_grupos_de_inv estigacion_2018.pdf

(5) Ordenanza 902 de 2016 - Por medio de la cual se adopta el Plan de Desarrollo del Departamento del Meta para el periodo 2016-2019: "Meta Tierra de Oportunidades. Inclusión, Reconciliación y Equidad". [Internet]. Gov.co. 2019 [modificado el 20 de agosto de 2020; citado el 19 de marzo de 2021]. Disponible en: http://www.asambleameta.gov.co/proyectos-deordenanzas/ordenanza-902-de-2016

(6) Según Ordenanza $\mathrm{N}^{\circ} 002$ del 31 de mayo de 2016, la Asamblea Departamental del Tolima, adopto el Plan de Desarrollo Departamental 2016-2019 "Soluciones que transforman". [Internet]. Gov.co. 2016 [citado el 19 de marzo de 2021]. Disponible en:https://www.tolima.gov.co/gobernacion /nosotros/organigrama/1271gobernacion/nuestros-planes/planes-dedesarrollo

(7) Oficina Asesora de Planeación Gobernación del Magdalena. Madgalena social: es la vía - Plan de Desarrollo 20162019 [Internet]. Santa Marta D.T.C.H., (Magdalena); 2016 [citado el 19 de marzo de 2021]. Disponible en: https://magdalena.micolombiadigital.gov.c o/sites/magdalena/content/files/000180/89 79_plandedesarrollomagdalena20162019. pdf

(8) Ordenanza 415 de 2016 - Por medio de la cual se adopta el Plan de Desarrollo Departamental del Valle del Cauca para el 
periodo 2016-2019: el Valle está en Vos. [Internet]. Gov.co. 2016 [citado el 19 de marzo de 2021]. Disponible en: http://crcvalle.org.co/wpcontent/uploads/Documentos/Plan-dedesarrollo-del-valle-del-cauca.pdf

(9) Ordenanza 012 de 2016 - Por medio de la cual se adopta el Plan de Desarrollo Departamental de Santander para el periodo 2016-2019: Santander nos Une. [Internet]. Gov.co. 2016 [citado el 19 de marzo de 2021]. Disponible en: https://www.santander.gov.co/index.php/g obernacion/documentacion/send/687-plande-desarrollo-departamental/4275ordenanza-012-de-2016-pdd-santandernos-une-2016-2019

(10) Ordenanza 006 de 2016 - Por medio de la cual se adopta el Plan de Desarrollo Departamental de Cundinamarca para el periodo 2016-2020: Unidos podemos más. [Internet]. Gov.co. 2016 [citado el 19 de marzo de 2021]. Disponible en: http://www.cundinamarca.gov.co/wcm/co nnect/10ae7a7a-7f25-43b1-899145001b4d7472/Plan_de_Desarrollo_2016 _2020.pdf?MOD=AJPERES\&CVID=lWr $1 \mathrm{uT} 3 \& \mathrm{CVID}=1 \mathrm{Wr} 1 \mathrm{uT} 3$.

(11) Ordenanza 011 de 2016 - Por medio de la cual se adopta el Plan de Desarrollo Departamental de Antioquia para el periodo 2016-2019: Antioquia piensa en grande. [Internet]. Gov.co. 2016 [citado el 19 de marzo de 2021]. Disponible en: https://www.asambleadeantioquia.gov.co/ $? \mathrm{p}=2213$

(12) Ordenanza 012 de 2016 - Por medio de la cual se adopta el Plan de Desarrollo Departamental de Nariño para el periodo 2016-2019: Nariño corazón del mundo. [Internet]. Gov.co. 2016 [citado el 19 de marzo de 2021]. Disponible en: https://publicadministration.un.org/unpsa/ Portals/0/UNPSA_Submitted_Docs/Plan\% 20de\%20Desarrollo\%20Departamental\%2 0Nari\%C3\%B1o\%20Coraz\%C3\%B3n\%2 0del\%20Mundo..pdf?ver=2018-11-29171310-447

(13) Ordenanza 007 de 2016 - Por medio de la cual se adopta el Plan de Desarrollo Departamental de Arauca para el periodo 2016-2019:Humanizando el desarrollo. [Internet]. Gov.co. 2016 [citado el 19 de marzo de 2021]. Disponible en: https://www.arauca.gov.co/gobernacion/n ormatividad/ordenanzas/3063-ordenanzan-07e-de-2016/file .

(14) Universidad Cooperativa de Colombia. Grupos y Investigación [Internet]. Investigación. [citado el 19 de marzo de 2021]. Disponible en: https://www.ucc.edu.co/investigacion/Pagi nas/grupos.aspx.

(15) Suárez-Hernández C. Orientación de la fase preliminar de togaf para su aplicación en proyectos de arquitectura [Tesis de Maestría]. Bucaramanga: Universidad Autónoma de Bucaramanga; 2014.

(16) CIO@GOV. El camino hacia un gobierno integrado [Internet]. Gov.co. [citado el 19 de marzo de 2021]. Disponible en: https://www.mintic.gov.co/gestionti/615/a rticles-5322_Revista_pdf.pdf

(17) Ávila-Correa BL. Modelos de Referencia de Arquitectura Empresarial para la Industria de Educación Superior. Rev Kill Téc. 2018;2(1):27-34. https://doi.org/10.26871/killkana_tecnica. v2i1.288.

(18) Granja-Cedeño C, Vallejo-Cedeño R. Adopción de un marco metodológico de arquitectura empresarial en una empresa 
gubernamental. Caso: De estudio administración de impuestos [Tesis de Maestría]. Quito: Pontificia Universidad Católica de Ecuador - PUCE; 2015.

(19) Ávila-Correa BL. Formulación de visionamiento estratégico de arquitectura empresarial aplicado a entidades de educación superior: un caso de aplicación [Tesis de Maestría]. Quito: Universidad de las Américas; 2016.

(20). Josey A. TOGAF® Versión 9.1 - Guía de Bolsillo [Internet]. Van Haren Publishing, editor. Zaltbommel, Netherlands: van Haren Publishing; 2013 [citado el 19 de marzo de 2021]. Disponible en: https://www.vanharen.store/togafregversion-91-guia-de-bolsillo/

(21) Santiago-Cely CP. Propuesta de Arquitectura Empresarial para una universidad como un apoyo a su desarrollo frente a los retos del Siglo XXI. In: 12th LACCEI Latin American and Caribbean Conference for Engineering and Technology (LACCEI'2014) [Internet]. Guayaquil, Ecuador: ESCUELA SUPERIOR POLITÉCNICA DEL LITORAL (ESPOL); 2014. p. 1-11. Disponible en: http://www.laccei.org/LACCEI2014Guayaquil/RefereedPapers/RP130.pdf.

(22) Sánchez LM, Villamil Sierra JM. Plan Piloto de Implementación del Modelo de Referencia de Arquitectura Empresarial Togaf 9.1 para la Dirección de Bienestar Universitario de la Universidad Nacional de Colombia Sede Bogotá. [Tesis de especialización]. Bogotá D.C.: Universidad Distrital Francisco José de Caldas; 2017.

(23) Contreras WMR, Delgado M del PS, Silva WGG. Diseño de un Modelo de Arquitectura Empresarial para el Macroproceso de Gestión Académica de la Universidad de Pamplona. RCTA. Tecnologías de Avanzada. 2017;2(26):805.

http://dx.doi.org/10.24054/16927257.v26. n26.2015.2384.

(24) Saboya Ríos N, Loaiza Jara OL, Lévano Rodríguez D. Diseño de un modelo de arquitectura empresarial para publicaciones científicas basado en ADM TOGAF 9.0. Apunt Univ. 2019;8(1):52-67. Disponible en: https://doi.org/10.17162/au.v8i1.179.

(25) Manual para el diseño y la construcción de indicadores. Instrumentos principales para el monitoreo de programas sociales de México [Internet]. Org.mx. 2013 [citado el 20 de marzo de 2021]. Disponible en: https://www.coneval.org.mx/InformesPubl icaciones/Paginas/Mosaicos/Manual-parael-diseno-y-la-construccion-deindicadores.aspx

(26). Universidad Cooperativa de Colombia. Misión y Visión [Internet]. Institución. [citado el 19 de marzo de 2021]. Disponible en:

https://www.ucc.edu.co/institucion/Pagina s/mision-vision.aspx. 